\title{
Turbulent heating of coronal active regions
}

\author{
Daniel O. Gómez† and Pablo Dmitruk \\ Department of Physics, Facultad de Ciencias Exactas y Naturales, \\ Ciudad Universitaria, (1428) Buenos Aires, Argentina \\ email: dgomez@df.uba.ar
}

\begin{abstract}
Magnetohydrodynamic turbulence has been proposed as a mechanism for the heating of coronal active regions, and has therefore been actively investigated in recent years. According to this scenario, a turbulent regime is driven by footpoint motions. The energy being pumped this way into active region loops, is efficiently transferred to small scales due to a direct energy cascade. The ensuing generation of fine scale structures, which is a natural outcome of turbulent regimes, helps to enhance the dissipation of either waves or DC currents.

We present an updated overview of recent results on turbulent coronal heating. To illustrate this theoretical scenario, we simulate the internal dynamics of a coronal loop within the reduced MHD approximation. The application of a stationary velocity field at the photospheric boundary leads to a turbulent stationary regime after several photospheric turnover times. This regime is characterized by a broadband power spectrum and energy dissipation rate levels compatible with the heating requirements of active region loops. Also, the energy dissipation rate displays a complex superposition of impulsive events, which we associate to the so-called nanoflares. A statistical analysis yields a power law distribution as a function of their energies, which is consistent with those obtained from observations. We also study the distributions of peak dissipation rate and duration of these events.
\end{abstract}

Keywords. solar corona, coronal heating, mhd turbulence, nanoflares.

\section{Introduction}

Models of coronal heating in loops have been traditionally classified into two broad categories, according to the time scales involved in the driving at the loop bases: (a) AC or wave models, for which the energy is provided by waves at the Sun's photosphere, with timescales much faster than the time it takes an Alfven wave to cross the loop; (b) DC or stress models, which assume that energy dissipation takes place by magnetic stresses driven by slow footpoint motions (compared to the Alfven wave crossing time) at the Sun's photosphere.

These scenarios seem mutually exclusive. Nevertheless two common factors prevail: (i) the source for the heating is the kinetic energy of the photospheric velocity field, (ii) the existence of fine scale structure is essential to speed up the dissipation mechanisms invoked.

Review articles on coronal heating (Narain \& Ulmschneider (1990), Narain \& Ulmschneider (1996), Gómez (1990), Zirker (1993)) explore the theoretical models in further detail. More recent reviews can be found in Mandrini et al. (2000), Demoulin et al. (2003), Aschwanden (2004), where observational tests of the models are also described.

A natural candidate for the dissipation of the energy provided by the photospheric motions is Joule heating, but the typical time scale to dissipate coronal magnetic stresses at the length scale of the driving motions is exceedingly long. This time scale can be estimated as $l^{2} / \eta \approx 10^{6}$ years ( $l$ is a typical length scale an $\eta$ is the plasma resistivity).

$\dagger$ Also at the Instituto de Astronomía y Física del Espacio, (1428) Buenos Aires, Argentina. 
Most of the theories of coronal heating invoke different mechanisms to speed up the energy dissipation (Parker (1972), Parker (1988), Heyvaerts \& Priest (1983), van Ballegooijen (1996), Mikić et al. (1989), Longcope \& Sudan (1994), Hendrix \& van Hoven (1996), Galsgaard \& Nordlund (1996), Gudiksen \& Nordlund (2002)).

\section{The case for turbulent heating}

One of the proposed scenarios to speed up dissipation is the assumption that the magnetic and velocity fields of the coronal plasma are in a turbulent state (Gómez \& Ferro Fontán (1988), Gómez \& Ferro Fontán (1992), Heyvaerts \& Priest (1992)). On these models, turbulent fluctuations of both the velocity and the magnetic fields are predominantly in the direction perpendicular to the main magnetic field. In a turbulent regime, energy is transferred from photospheric motions to the magnetic field and then cascades toward small scales due to nonlinear interactions, until highly structured electric currents are formed. The development of fine scales to enhance the dissipation of either waves or DC currents is a natural outcome of turbulence models.

Direct numerical simulations of the turbulent regime of magnetic and velocity fields in a coronal loop has been performed in Einaudi et al. (1996), Dmitruk \& Gómez (1997), Dmitruk et al. (1998), Georgoulis et al. (1998) where a two-dimensional MHD model is used to imitate the dynamics of transverse slices of a loop. A distinguishing characteristic of these studies is the bursty nature of the energy dissipation. The peaks of the energy dissipation time series of the numerical simulations results have been associated with energy dissipation events in the corona. The relatively low energy content of these individual events suggests an association with the so-called nanoflare heating scenario (see Parker (1972), Parker (1988)), as opposed to larger energy events, such as flares and microflares. The distribution of energy events (histograms) obtained from the numerical simulations is of a power-law type for a broad range of energies in the nanoflare energy region, from $10^{23}$ erg to $10^{27} \mathrm{erg}$. There are recent observations Aschwanden \& Parnell (2002), Benz \& Krucker (2002) that seem to confirm the power-law behavior of the energy distributions, although the indirect nature of the observational data at this range and the differences in statistical methods can not provide a definite value of the power-law index at this point. At larger energies (microflare and flare regions) there is compelling observational evidence indicating power laws with indices of about 1.5-1.8 (Hudson (1991), Crosby et al. (1993), Shimizu (1995)).

More recent numerical studies of turbulent regimes in coronal loops have been performed using a three-dimensional reduced MHD (RMHD) approximation, such as those in Dmitruk \& Gómez (1999), Watkins et al. (2001), Dmitruk et al. (2003), Rappazzo et al. (2007). In some of these studies the focus is on aspects such as the dissipation rate and the energy spectra, as well as on their dependence on an important parameter of the dynamics of the loop, namely, the ratio between the photospheric turnover time $t_{p}$ and the Alfven crossing time of the loop $t_{A}$. Different turbulent regimes can be obtained as this time ratio is varied. The bursty nature of the energy dissipation series is also observed in these numerical studies, although the larger computational demand for three-dimensional studies poses a limitation to statistical studies of dissipative events.

An alternative and complementary line of research is to consider the so-called shellmodel approximation. These theoretical models drastically reduce the computational demand by replacing the spatial degrees of freedom with shells in wavevector space (see for instance Boffetta et al. (1999)). More recently, shell models have been developed for RMHD by by Nigro et al. (2004) and also by Buchlin \& Velli (2007). An alternative onedimensional MHD model is also presented in Galtier (1999). Because of this reduction in 


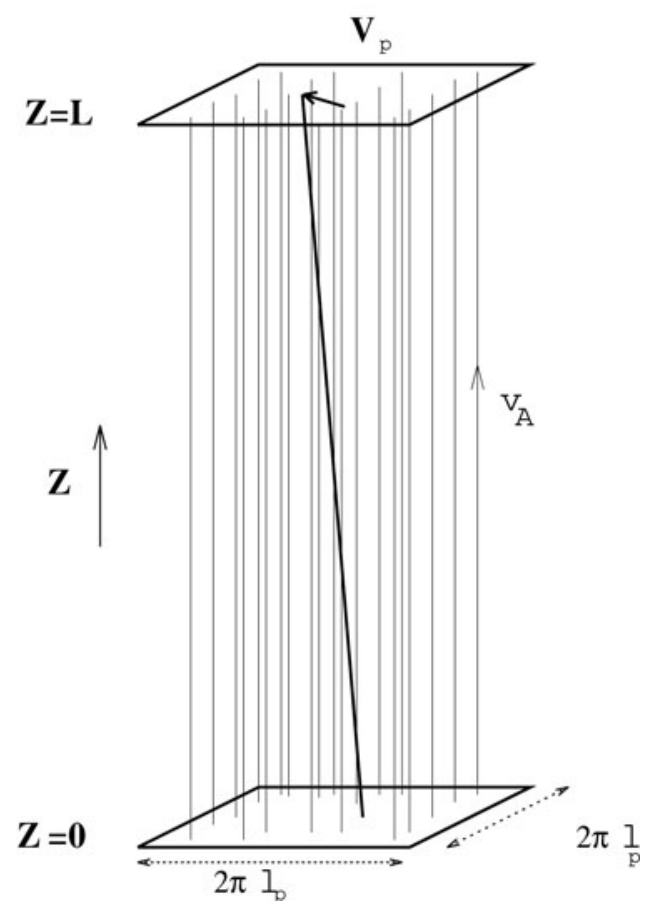

Figure 1. Cartoon model of a coronal loop. The planes $z=0$ and $z=L$ correspond to the photosphere.

computational load, shell-models allow much larger Reynolds numbers and longer time series, in comparison with the direct numerical simulations mentioned above. Of course, one important aspect that is missing in these models is the fine spatial structure, but in principle they seem to be reasonable anzats for the statistical study of dissipative events (provided the assumptions made in deriving the shell equations are akin to the fully developed turbulence regimes).

\section{Reduced MHD}

In what follows we focus on the theoretical description of a relatively homogeneous bundle of fieldlines. The loop footpoints are deeply rooted into the photosphere, which moves individual fieldlines around, thus generating magnetic stresses in the coronal portion of the loop. Under the assumption that a coronal loop reaches a stationary turbulent state, Milano et al. (1997) found that its response to a broad range of photospheric driving frequencies can be modeled by a closure model. At least for photospheric power spectra decreasing as power laws of both the wavenumber and the time frequency, the heating of the loop is dominated by DC heating.

We therefore consider a simplified model of a coronal magnetic loop with length $L$ and cross section $2 \pi l_{p} \times 2 \pi l_{p}$, where $l_{p}$ is the lengthscale of typical photospheric motions. For elongated loops, i.e. such that $2 \pi l_{p}<<L$, it is reasonable to neglect toroidal effects. The main magnetic field $\mathbf{B}_{\mathbf{0}}$ is assumed to be uniform and parallel to the axis of the loop (the $z$ axis). The planes at $z=0$ and $z=L$ correspond to the loop footpoints at the photosphere, as shown in Figure 1.

Under these assumptions, we are able to use the reduced MHD approximation Strauss (1976), in which the plasma moves incompressibly in planes perpendicular to the axial 
field $\mathbf{B}_{\mathbf{0}}$, and the transverse component of the magnetic field is small compared to $\mathbf{B}_{\mathbf{0}}$. The very high electric conductivity (frozen field) allows photospheric motions to easily drive magnetic stresses in the corona Parker (1972), the field lines twist and bend due to these motions and this generates tranverse components of velocity $\mathbf{u}$ and magnetic field b. Therefore:

$$
\begin{gathered}
\mathbf{B}=B_{0} \mathbf{z}+\mathbf{b}(x, y, z, t), \quad \mathbf{b} \cdot \mathbf{z}=0 \\
\mathbf{u}=\mathbf{u}(x, y, z, t), \quad \mathbf{u} \cdot \mathbf{z}=0
\end{gathered}
$$

Since both $\mathbf{b}$ and $\mathbf{u}$ are two-dimensional and divergence-free fields, they can be represented by scalar potentials:

$$
\begin{aligned}
& \mathbf{b}=\nabla \times(a \mathbf{z})=\nabla a(x, y, z, t) \times \mathbf{z} \\
& \mathbf{u}=\nabla \times(\psi \mathbf{z})=\nabla \psi(x, y, z, t) \times \mathbf{z}
\end{aligned}
$$

where $\nabla$ indicates derivatives in the $x, y$ plane.

The reduced MHD equations for the potentials $\psi$ and $a$ are Strauss (1976):

$$
\begin{gathered}
\partial_{t} a=v_{A} \partial_{z} \psi+[\psi, a]+\eta \nabla^{2} a \\
\partial_{t} w=v_{A} \partial_{z} j+[\psi, w]-[a, j]+\nu \nabla^{2} w
\end{gathered}
$$

where vorticity $w$ and current density $j$ relates to the potentials as:

$$
w=-\nabla^{2} \psi, j=-\nabla^{2} a
$$

The brackets $[A, B]=\partial_{x} A \partial_{y} B-\partial_{y} A \partial_{x} B$ are the standard Poisson brackets and $v_{A}=B_{0} / \sqrt{4 \pi \rho}$ is the Alfvén velocity ( $\rho$ is the plasma density).

Eqn (3.5) describes the advection of the potential $a$ and Eqn (3.6) corresponds to the evolution of vorticity $w$. The terms $v_{A} \partial_{z}$ represent the coupling between neighboring $z=$ constant planes. The $\nabla^{2}$ terms represent dissipative effects, the constants $\eta$ and $\nu$ being the resistivity and viscosity coefficients. The nonlinear terms are those represented by the Poisson brackets. Their role is to couple normal modes in such a way that energy, and other ideal invariants, can be transferred between them.

\section{RMHD turbulence}

We numerically explore the feasibility of a turbulent scenario for coronal loops, describing the internal dynamics of loops through the RMHD approximation. We assume periodicity for the lateral boundary conditions, and specify the velocity fields at the $z=0$ and $z=L$ photospheric boundaries. In particular, we assume

$$
\psi(z=0)=0, \quad \psi(z=L)=\Psi(x, y)
$$

where $\Psi(x, y)$ is the stream function which describes stationary and incompressible footpoint motions on the photospheric plane. The strength of this external velocity field is therefore proportional to a typical photospheric velocity $V_{p} \approx 1 \mathrm{~km} . \mathrm{s}^{-1}$. We model the photospheric boundary motion in Eqn (4.1) as $\Psi_{\mathrm{k}}=\Psi_{0}=$ constant inside the ring $3<l_{p}|k|<4$, and $\Psi_{\mathrm{k}}=0$ elsewhere, to simulate a stationary and isotropic pattern of photospheric granular motions of diameters between $2 \pi l_{p} / 4$ and $2 \pi l_{p} / 3$. The typical 

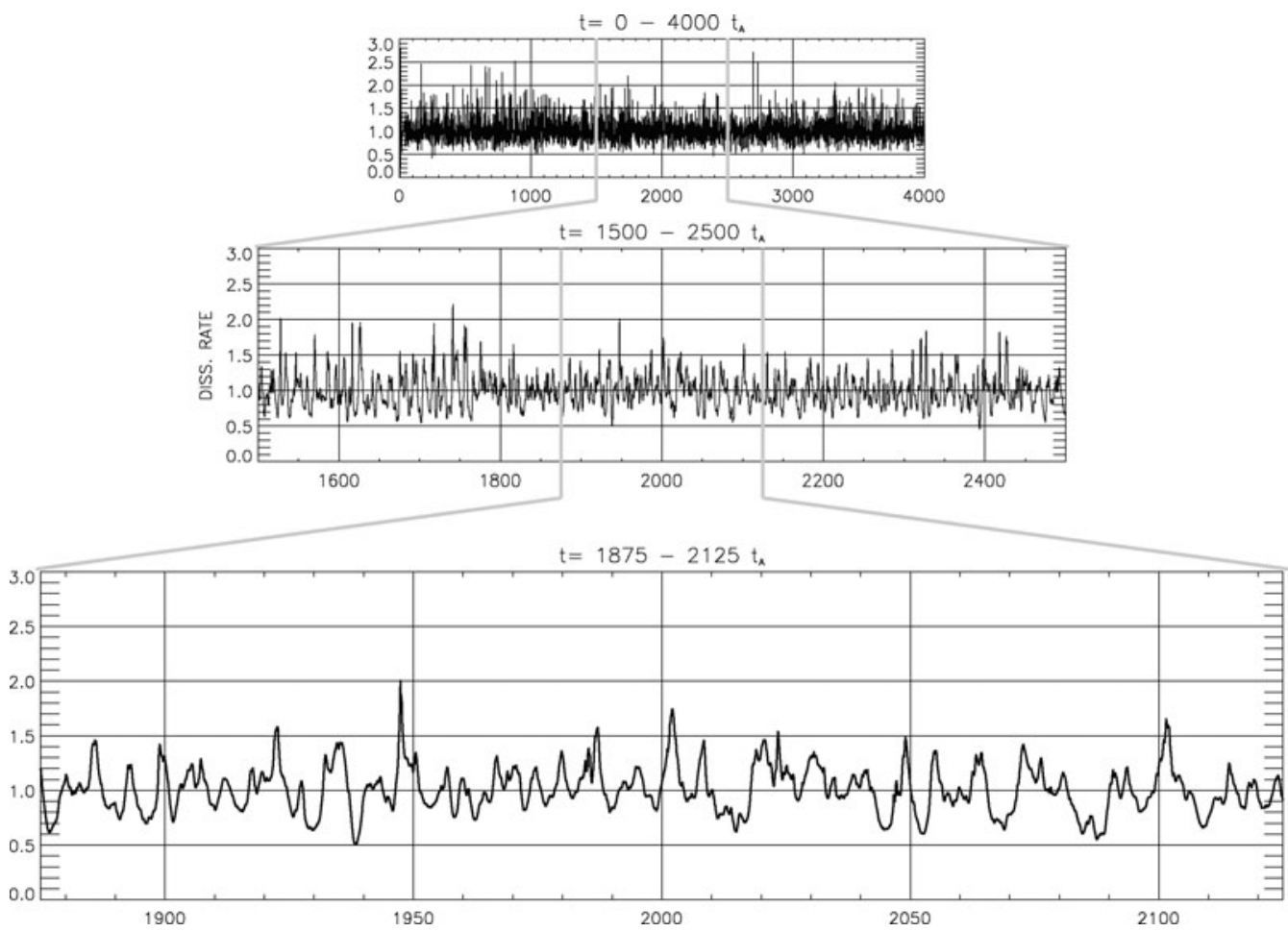

Figure 2. Energy dissipation rate vs. time. The upper panel shows the complete time series $\left([0-4000] t_{A}\right)$, the central panel blows up the central portion $\left([1500-3500] t_{A}\right)$, and the lower panel shows the range $\left([1875-2125] t_{A}\right)$.

timescale associated to these driving motions, is the eddy turnover time, which is defined as $t_{p}=l_{p} / V_{p} \approx 10^{3} \mathrm{sec}$. We chose a narrowband and non-random forcing to make sure that the broadband energy spectra and the signatures of intermittency that we obtained (see below) are exclusively determined by the nonlinear nature of the MHD equations.

To transform Eqs (3.5)-(3.6) into their dimensionless form, we choose $l_{p}$ and $L$ as the units for transverse and longitudinal distances $\left(l_{p} \approx 10^{3} \mathrm{~km}\right.$ and $\left.L \approx 10^{4}-10^{5} \mathrm{~km}\right)$ and $t_{A} \equiv L / v_{A}$ as the time unit $\left(t_{A} \approx 10-100 \mathrm{sec}\right)$.

In Figure 2 we show the energy dissipation rate obtained for a rather long $\left(4000 t_{A}\right)$ RMHD simulation. The upper panel shows the complete time series $\left([0-4000] t_{A}\right)$, while the panels below subsequently enlarge the central portion of the series to show its fine temporal structure. The spiky nature of this time series is caused by the ubiquitous presence of intermittency in turbulent regimes. As a working hypothesis, we tentatively associate each of this spikes of energy dissipation with Parker's nanoflares (see Parker (1988)). In the next section we study the statistical properties of these dissipation events.

Besides the intermittent features of the time series displayed in Fig. 2, a robust stationary regime can be observed, which is attained after a few photospheric turnover times. The dependence of this mean dissipation rate $\epsilon$ with the physical parameters of the loop is

$$
\epsilon \propto \frac{\rho l_{p}^{2}}{t_{A}^{3}}\left(\frac{t_{A}}{t_{p}}\right)^{\frac{3}{2}}
$$

(see Dmitruk \& Gómez (1999), and also Dmitruk \& Gómez (1997) for a similar two- 


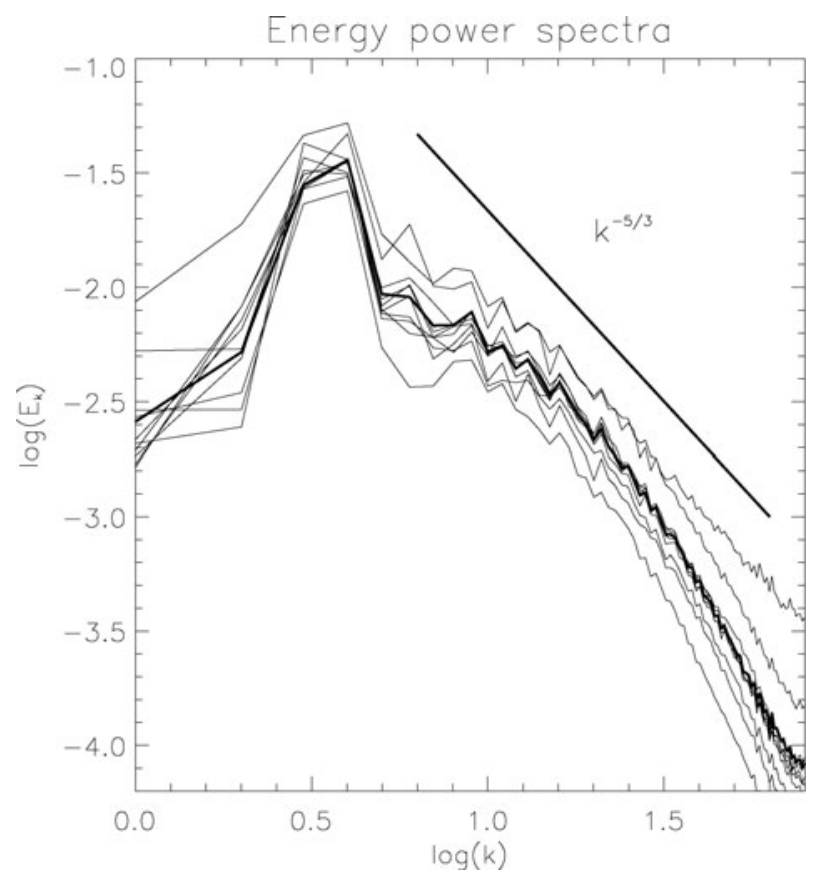

Figure 3. Energy power spectra for ten different times (separated by $\Delta t=100 t_{A}$ ) within the stationary regime. The thick trace is the time-averaged spectrum. The Kolmogorov slope is also displayed for reference.

dimensional study). The turbulent nature of this statistically stationary regime can be seen in Fig. 3, which shows energy power spectra taken at ten different times (separated by $\left.\Delta t=100 t_{A}\right)$. We also overlaid the time-averaged spectrum (thick trace) as a reference, as well as the $E_{k} \propto k^{-5 / 3}$ Kolmogorov slope. The numerical resolution of the RMHD simulations is $256 \times 256 \times 64$. This moderate resolution allows very long integration times, as needed for the statistical analysis described in Section 5. The kinetic and magnetic Reynolds numbers of our simulation $\left(R e=\frac{l_{p}^{2}}{\eta t_{A}}=R m\right.$ ) were carefully chosen to guarantee a proper resolution of the smallest spatial structures (i.e. the largest wavenumbers), where dissipation becomes dominant.

The spatial structures where most of the energy dissipation takes place are shown in Fig. 4, which displays the electric current density distribution in a transverse cut at half length of the loop. White (black) structures show concentrations of positive (negative) current density, which clearly resemble the transverse cut of a complex distribution of current sheets. This is precisely the case, as shown by the three-dimensional spatial distribution of Joule dissipation (i.e. $\left.\eta j^{2}\right)$ in Fig. fig:cube.

\section{Statistics of events}

Parker (1988) conjectured that the energy dissipation of the stressed magnetic structures takes place in a large number of small events, which he termed nanoflares. The superposition of a large number of such events would give the global appearance of a spatially homogeneous and stationary heating process.

From a turbulent scenario, it is straightforward to associate the spikes of energy dissipation discussed in $\S 4$ (and shown in Fig. 2), to Parker's nanoflares (Dmitruk \& Gómez (1997), Dmitruk et al. (1998)). We draw a horizontal line in the time series of energy 


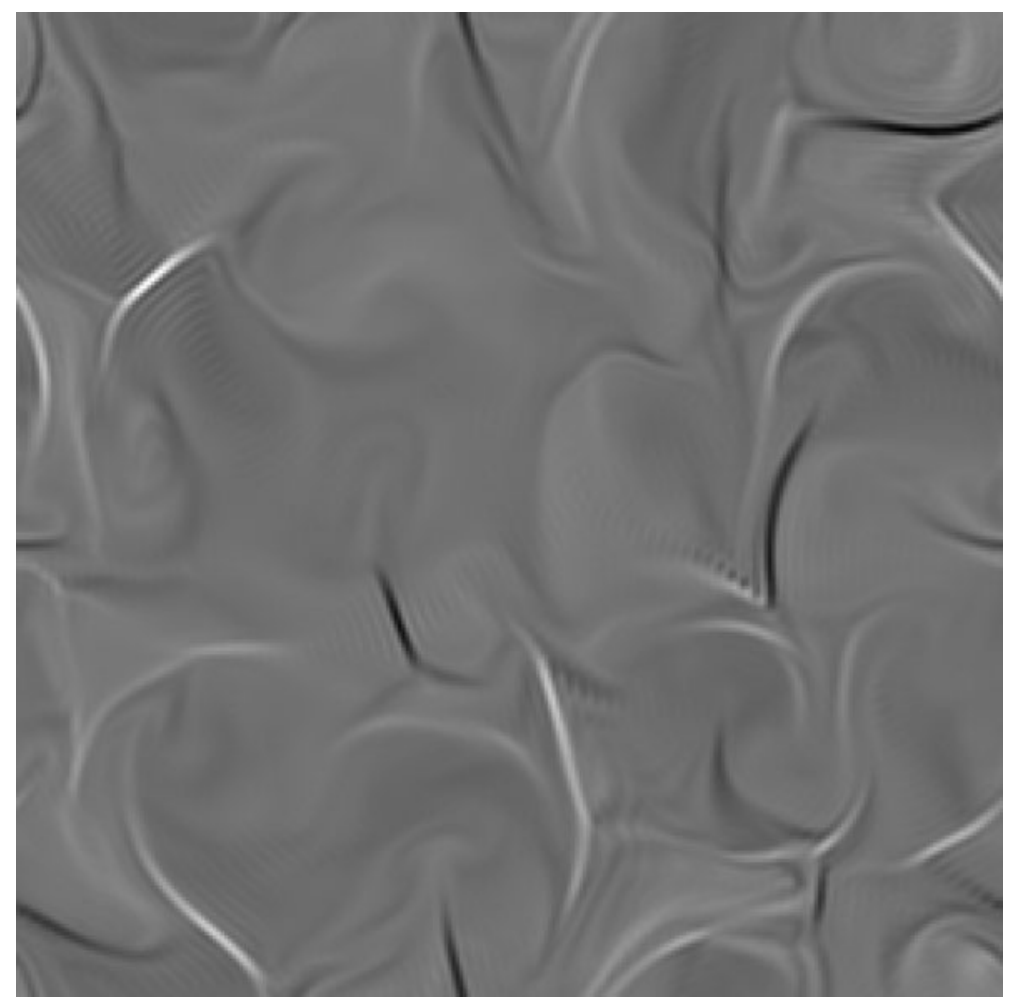

Figure 4. A transverse cut at half length of the loop shows the spatial distribution of current density. Upflowing current is indicate in white, and downflowing current is shown in black.

dissipation rate (Fig. 2) somewhere above its mean value, and define every structure above this threshold as a dissipation event. The influence of the definition of an event on the statistics is discussed in Buchlin et al. (2005). We accumulate these events, and perform a statistics of properties such as their total dissipated energies $E$, their peak dissipation rates $P$, and their durations $T$. The corresponding histograms, i.e. number of events with energies (peak dissipation rates, durations) in the range $[E, E+d E]([P, P+d P]$, $[T, T+d T])$, are shown in Fig. 6 .

The physically meaningful slope is the one displayed toward large values, i.e. toward the right side of each panel. The distribution of energies displays a power law

$$
N(E) d E \propto E^{-1.5} d E
$$

We estimate a $10 \%$ error in the determination of this slope. Power laws with indices close to -2 are obtained both for the distribution of peak dissipation rate and duration. We also performed correlation analysis of these quantities, and obtain that $T \propto P \propto E^{1 / 2}$.

The distribution toward small values, i.e. toward the left side of each panel, are contaminated by the arbitrary choice of the threshold defining our dissipation events. For the histograms shown in Fig. 6 , the threshold was set $1 \sigma$ above the mean value of the time series. It is straightforward to show that for the smallest events, the energy distribution should display a power law $E^{-1 / 2}$, while for the peak dissipation rate and duration, the slope should be close to zero. 


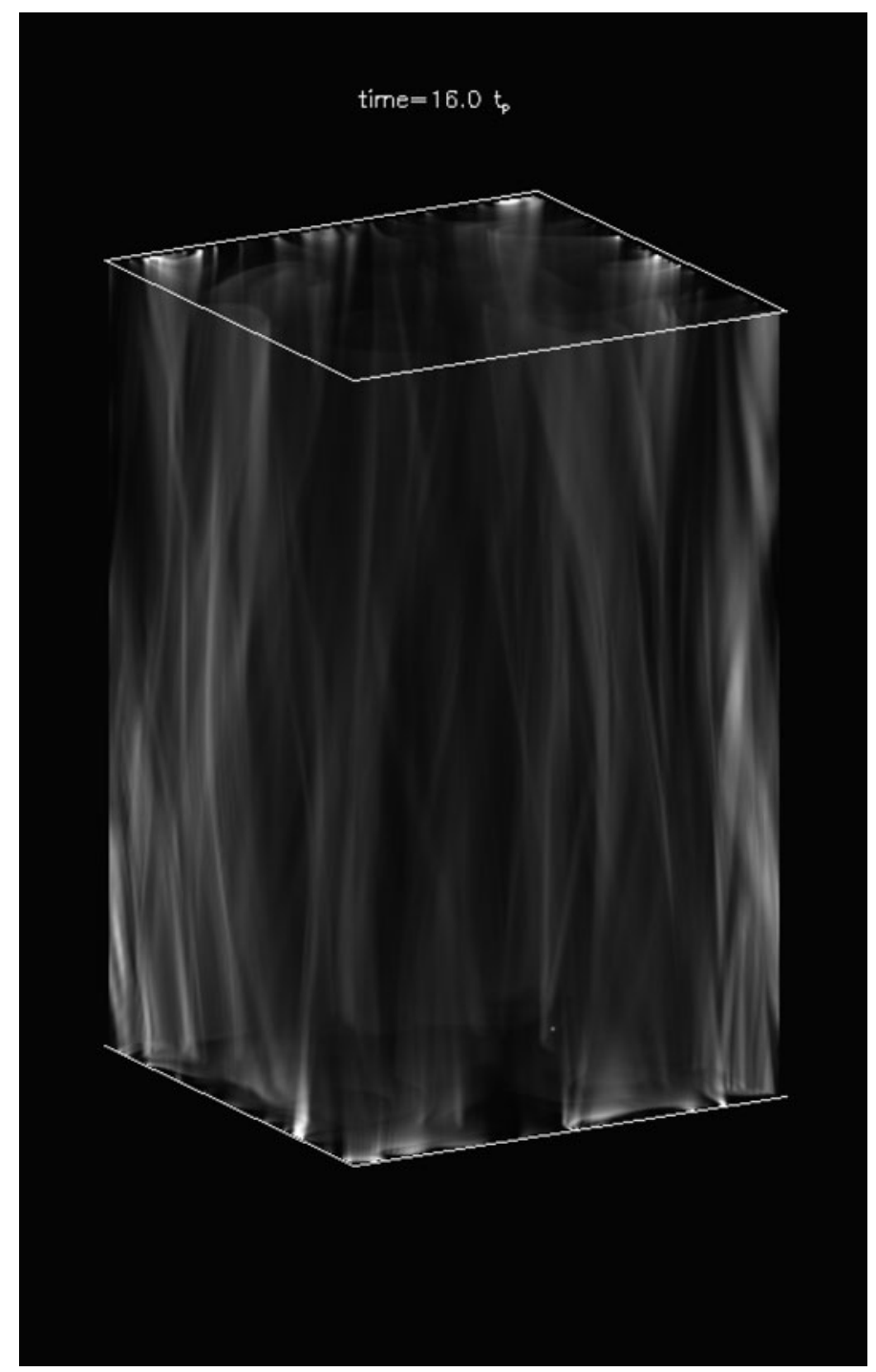

Figure 5. The spatial distribution of the energy dissipation rate at $t=16 t_{p}$, well within the stationary turbulent regime.

The events reported here span the energy range from $E_{\min } \simeq 10^{25}$ erg to $E_{\max } \simeq$ $3 \times 10^{26} \mathrm{erg}$, for typical values of the loop parameters. These slopes are consistent with those derived from various observational studies and reported by Aschwanden (2004).

\section{Conclusions}

The magnetohydrodynamic turbulence scenario as a promising mechanism for coronal heating in loops is briefly reviewed. In particular, we discuss the role of MHD turbulence in developing small scales and in speeding up energy dissipation by the ensuing direct energy cascade. MHD turbulence, once developed, enhances dissipation of both AC and DC structures.

Recent results of RMHD numerical simulations of the internal dynamics of a coronal loop are also shown. A turbulent stationary regime is achieved after several photospheric 

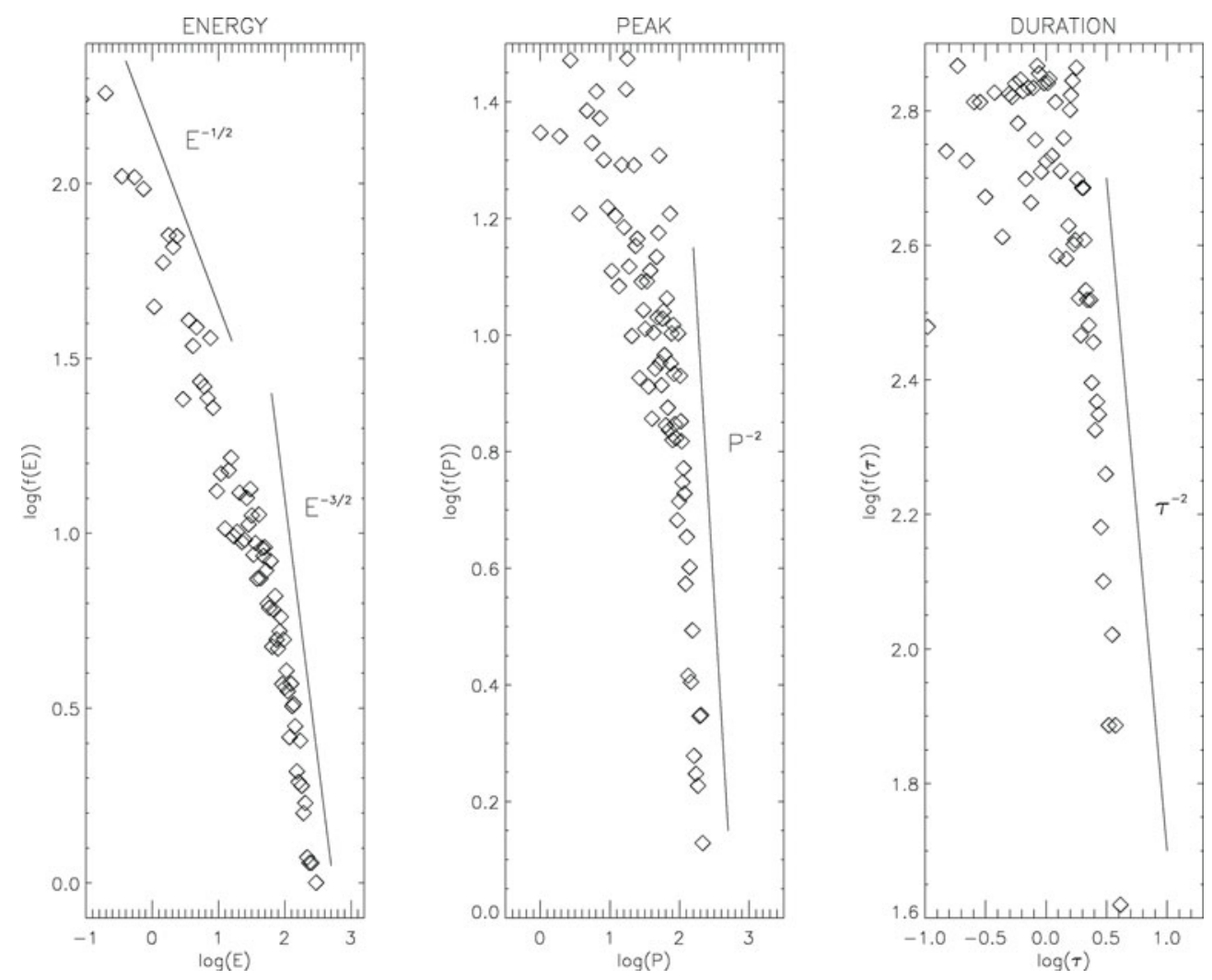

Figure 6. Shows the histograms for energy, peak dissipation rate and duration of the recorded events. The threshold was chosen $1 \sigma$ above the mean value. Aproximate slopes are also displayed for reference.

turnover times, as a result of the action of a stationary velocity field pattern at the photospheric boundary. A Kolmogorov-like broadband energy spectrum is obtained. The spatial distribution of energy dissipation shows the concentration of the electric currents in structures that resemble a complex distribution of current sheets. The obtained heating rate levels are compatible with the requirements for coronal active regions.

The energy dissipation time series are of a bursty nature and therefore we associate individual energy dissipation events to the so-called nanoflares, widely discussed in the literature. A statistical analysis of these events shows power law distributions for their energies, peak dissipation rates and durations. The slopes derived for these power laws are fully consistent with previous studies performed within a two-dimensional MHD approximation. They are also consistent with the various recent observational studies reported in the literature.

\section{Acknowledgements}

This research is supported by the Agencia Nacional de Promoción de Ciencia y Tecnología under grant PICT2005/33370 to IAFE. DOG and PD are members of the Carrera del Investigador Científico of CONICET. 


\section{References}

Aschwanden, M.J. 2004, in Physics of the Solar Corona. An Introduction, Springer-Verlag, Berlin.

Aschwanden, M.J., \& Parnell, C.E. 2002, ApJ, 572, 1048.

Benz, A.O., \& Krucker, S. 2002, ApJ 568, 412.

Boffetta, G., Carbone, V., Giuliani, P., Veltri, P., \& Vulpiani, A. 1999, Phys. Rev. Lett., 83, 4662.

Buchlin, E., Galtier, S., \& Velli, M. 2005, Astron. Astrophys., 436, 355.

Buchlin, E., \& Velli, M. 2007, ApJ, 662, 701.

Crosby, N.B., Aschwanden, M.J., \& Dennis, B.R. 1993, Solar Phys., 143, 275.

Demoulin, P., van Driel-Gesztelyi, L., Mandrini, C.H., Klimchuk, J.A., \& Harra, L. 2003, ApJ, 586,592 .

Dmitruk, P., \& Gómez, D.O. 1997, ApJ, 484, L83.

Dmitruk, P., Gómez, D.O., \& DeLuca, E. 1998, ApJ, 505, 974.

Dmitruk, P., \& Gómez, D.O. 1999, ApJ, 527, L63.

Dmitruk, P., Gómez, D.O., \& Matthaeus, W.H. 2003, Phys. Plasmas, 10, 3584.

Einaudi, G., Velli, M., Politano, H., \& Pouquet, A. 1996, ApJ, 457, L113.

Galsgaard, K., \& Nordlund, A. 1996, J. Geophys. Res., 101, 13445.

Galtier, S. 1999, ApJ, 521, 483.

Georgoulis, M., Velli, M., \& Einaudi, G. 1998, ApJ, 497, 957.

Gómez, D.O. 1990, Fund. Cosmic Phys., 14, 361.

Gómez, D.O., \& Ferro Fontán, C. 1988, Solar Phys., 11633.

Gómez, D.O., \& Ferro Fontán, C. 1992, ApJ, 394, 662.

Gudiksen, B.V., \& Nordlund, A. 2002, ApJ, 572, L113.

Hendrix, D.L., \& van Hoven, G. 1996, ApJ, 467, 887.

Heyvaerts, J., \& Priest, E.R. 1983, Astron. Astrophys., 117, 220.

Heyvaerts, J., \& Priest, E.R. 1992, ApJ, 390, 297.

Hudson, H.S. 1991, Solar Phys., 133, 357.

Longcope, D.W., \& Sudan, R.N. 1994, ApJ, 437, 491.

Mandrini, C.H., Demoulin, P., \& Klimchuk, J.A. 2000, ApJ, 530, 999.

Mikić, Z., Schnack, D.D., \& van Hoven, G. 1989, ApJ, 338, 1148.

Milano, L.J., Gómez, D.O., \& Martens, P.C.H. 1997, ApJ, 490, 442

Narain, U., \& Ulmschneider, P. 1990, Space Sci. Rev., 54, 377.

Narain, U., \& Ulmschneider, P. 1996, Space Sci. Rev., 75, 453.

Nigro, G., Malara, F., Carbone, V., \& Veltri, P. 2004, Phys. Rev. Lett., 92, 194501.

Parker, E.N. 1972, ApJ, 174, 499.

Parker, E.N. 1988, ApJ, 330, 474.

Rappazzo, A.F., Velli, M., Einaudi, G., \& Dahlburg, R.B. 2007, ApJ, 657, L47.

Shimizu, T. 1995, Publ Astr. Soc. Japan, 47, 251.

Strauss, H. 1976, Phys. Fluids, 19, 134

van Ballegooijen, A.A. 1986, ApJ, 311, 1001.

Watkins, N.W., Oughton, S., \& Freeman, M.P. 2001, Planetary and Space Sci., 49, 1233.

Zirker, J.B. 1993, Solar Phys., 148, 43. 